\title{
Lessons from ICT Design at a Healthcare Worker-Centered System for a Chronic Mental Care Hospital
}

\author{
Junia Anacleto \\ UFSCar \\ Brazil \\ junia@dc.ufscar.br
}

\author{
Sidney Fels \\ UBC \\ Canada \\ ssfels@ece.ubc.ca
}

\author{
Roberto Calderon \\ UBC \\ Canada \\ roberto@robertocalderon.ca
}

\begin{abstract}
We report on our research on designing natural ICT tools and solutions for integration into a non-ICT based workflow at a Brazilian chronic care hospital. Our healthcare worker-centered approach for continuous lifecare contrasts from the more typical acute care situations where systems are geared towards a combination of patientcentered or administrator-centered designs. While acute care patients tend to spend a relatively short period of time in the hospital with life and death situations, chronic mental care focuses on improving a patient's life quality as they age. Thus, the therapeutic processes are geared towards collaboratively preparing patients to be able to establish a routine for what is defined as an independent normal life in their community. We report on three main findings from our worker-centered design in this environment: 1 . Supporting workers communication and workflow using an online social intranet is effective, 2. Designing for appropriation as well as adoption is important and 3. Public workflow visualization can influence workers behavior, according to their cultural values. The approach we suggest may apply to other long-term assistant scenarios such as nursing homes, care for people with disabilities and teaching and parenting contexts.
\end{abstract}

\section{Author Keywords}

Worker-centered design, appropriation and adoption, online social intranet, chronic mental care, long-term care, cultural behaviour.

\section{ACM Classification Keywords}

H.5.2 User Interfaces (Theory and methods), H.4.1 Office

Automation (Workflow management)

\section{INTRODUCTION}

Long-term care in contexts of mental health, disabilities and aging are emerging as a fact of life for many of the world's countries. In these contexts, often the person being cared for only receives a quality of care that depends upon the quality of treatment and procedures of the care workers. Our research is looking at these healthcare workers by focusing on their complex workflows to design applications and systems to integrate into their existing natural collaborative practices [2,3], considering their cultural values that shapes their relationship.
At the moment, we have partnered with the Brazilian Centre for Integral Health Support (CAIS) Clemente Ferreira [4], in Brazil. This is a special chronic care center for individuals with neurological and brain disorders. While the primary objective of the Center is for professional health staff to facilitate patients' transitioning from the center back into normal society and fitted to the local culture, in reality, most patients live until they die at the institution. The center has 3 floors with 6 wings with 600 patients and 800 professionals distributed throughout. The center has four distinct professional roles: administration, health professionals, nurse assistants and maintenance. Currently the Center has essentially no Wi-Fi or cell phone coverage due to architectural issues (at least $70 \mathrm{~cm}$ thick concrete walls) and minimal IT budget (no funds to install Wi-Fi service for professionals). They use voice and a paper-based system for their primary workflow mechanisms that are the basis for that cultural ecosystem. While there are rooms for the healthcare workers to work in and write notes, i.e. nursing stations, most of their time is spent moving around the center attending to daily routines.

For our research we have been focusing both on mobile natural solutions as well as natural user interfaces in a developing economy countries. This is in contrast to looking at Center information system based around desktop computing and GUI based interaction. Workers in developing economies countries are appropriating their personal consumer grade mobile devices at work substantially faster than their developed economy counterparts [14], so that the BYOD philosophy is brought up by the users and not by the organization, becoming part of that culture. Thus, studying these groups and working on new design strategies with them will illuminate the pathway forward for the next wave of ICT in work environments that are not dependent on existing legacy systems as we see in developed countries.

In this paper, we describe three main findings from our research at the Center targeted towards the health professionals and not the patients or administrators. The three main findings are: 1.Supporting worker social and professional behaviour based on communication and workflow using an online social intranet is effective, 2.Designing for cultural based values appropriation as well 


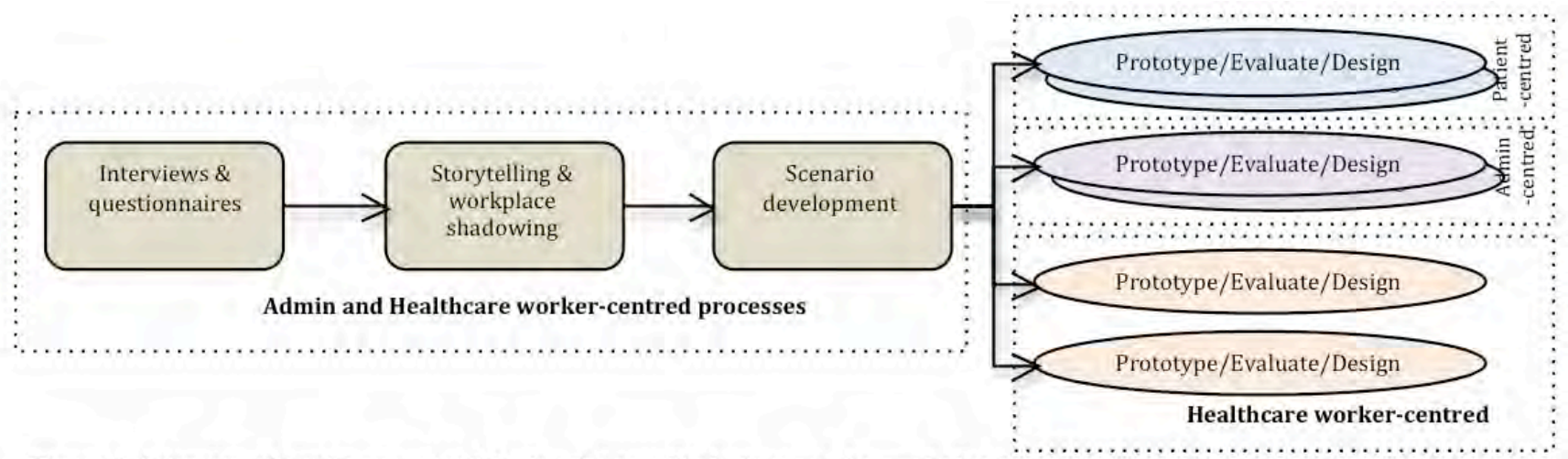

Figure 1: Diagram of our User-centred Design Process Evolution: we observed that for chronic care, the healthcare worker centred designs were critical for introducing technology.

as adoption is important and 3.Public workflow visualization can influence worker behavior considering cultural values. We believe that working with these professionals is an opportunity to design information technology based workflow that avoids a Windows, Icons, Menus and Pointers (WIMP) oriented strategy [3]. Likewise, we can use their workflow as a starting point in deciding what natural is for that cultural ecosystem, given that their workflow has evolved over more than two decades of practice [2].

\section{RELATED WORK}

In general, e-Health has been oriented around the use of the internet and other information and communication technologies (ICT) to improve access, efficiency, effectiveness and quality of clinical processes used by health organizations, patients and consumers in an attempt to improve the health of patients [6]. Much of the work for e-Health has targeted acute care situations, as proper functioning can be the matter of life and death. These systems tend to be either patient-oriented (electronic health records, aids for professional decision making) or administrator oriented (better tracking of costs and care systems for decision making). For example, in the works of [15][9][1][27], work focuses on aspects of the patient experience, providing better feedback to patients, helping with patient motivation or improving a therapeutic process. Likewise, there are a plethora of hospital information systems with a long history [28] as the promise of ICT technology to improve hospital workflows, patient record management and patient outcomes has yet to be fully realized. However, these are more oriented towards the administration and management of information flow, including patient data, throughout a hospital. We differentiate our approach from these acute healthcare systems that tend to be centered on either patients or administration of the hospital.

Our research approach follows the path of many studies $[23,11]$ that have been carried out in the application of ICT within hospital settings with many different objectives, like: improving communication processes among hospital staff, providing more accurate diagnostic tools and treatments, assist in therapy processes, increase patient's medication adherence, and others. Typically, a user design processes (UCD) approach [20] is adapted from human computer interaction and software engineering approaches including: interviews as a starting point $[21,25]$ and considering enduser participation in the design process [21,25,16,22], creation and validation of scenarios [21,25,22] added to storyboarding $[25,13,26]$ and a short development cycle of prototypes from low to high fidelity $[22,25,16]$ being evaluated and redesigned.

\section{A HEALTHCARE WORKER-CENTRED DESIGN}

Our user centered design (UCD) process has been ongoing since June 15, 2011 to now. We have had 15 half-day or full day meetings with healthcare staff, documented and validated their workflow, established 4 scenarios, and created 3 prototypes for testing, including a long term study that is ongoing. As well, we have applied a survey and a one week workplace shadowing activity. The UCD has

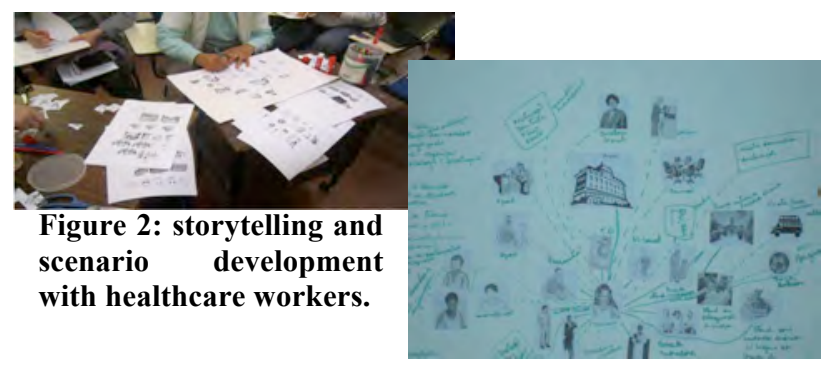

focused on all three main aspects of the Center, administrator requirements, healthcare worker requirements and patient requirements. Figure 1 illustrates how our research activities progressed. As we progressed through the patient and administrator centered prototypes (blue and purple), we discovered that many of the activities in the Center that help to maintain the well-being of the patients have very little to do with direct patient intervention or administrative duties. Instead, they are concerned with maintaining a copacetic workplace with an easy flow of dialogue, for social and professional interaction, strengthening the cultural ecosystem amongst professionals 
and patients. In this context, the Center runs more like a family home where the routine of daily life is accentuated by regular stimulating cultural and social events that are created coupled to pharmaceutical interventions for the residents to mitigate behavioral difficulties that either endanger a resident or disrupt the daily flow of the residents. It is from this observation that we created two prototypes to focus on how the healthcare workers establish a reasonable quality of life for the residents. The first prototype, CareHub, shown in figure 3, is oriented towards personal daily routine and digital asset management. The second prototype, shown in figures 5-8, is a simple ticketing system we used to investigate whether public visualization of 'favors' could impact the work environment.

During our storyboarding processes for scenario development, we developed an understanding about the various routine activities that the healthcare worker does. Figure 2 shows an image from one of the exercises we did during the storytelling activities. Some of the activities include checking on residents, giving medication, taking residents to social activities, washing, feeding and making notes about the residents. During the workplace shadowing, we also noted that they do many "favors" for each other and that forms a particularly important part of how jobs gets done on a regular basis. These "favors" transcend the official hierarchy of duties at the center. As well, much time is spent talking to each other about residents and activities. Finally, we also noticed on our regular visits that essentially every week there is some "special" event organized for the residence to meet and spend time together in a social setting.

\section{CAREHUB: A SOCIAL INTRANET FOR SUPPORTING WORKER ACTIVITY}

Based on our observations and the participatory design, we developed a prototype that we call CareHub [24]. The CareHub design distributes content between workers' smartphones and tablets and large screen displays intended to be distributed throughout the center. The details of content distribution are based on the discussion with the staff. Specifically, the smartphones are for referencing private information with minimal editing, including checking agenda, looking at patient information and messages. The tablets are designed to support management of digital assets such as photos and videos, agendas, patient files and notes, game configurations and activation menus, a general bulleting board and a birthday/event list. In contrast to the smartphone, the tablet is intended to be the primary place for data editing and entry. The mobile platforms link to the large displays. The large displays show content that we determined from discussions that would be appropriate for public viewing. In particular, full screen view of photos, videos and video games for residents to play, birthday announcements and a bulletin board. Figure 3

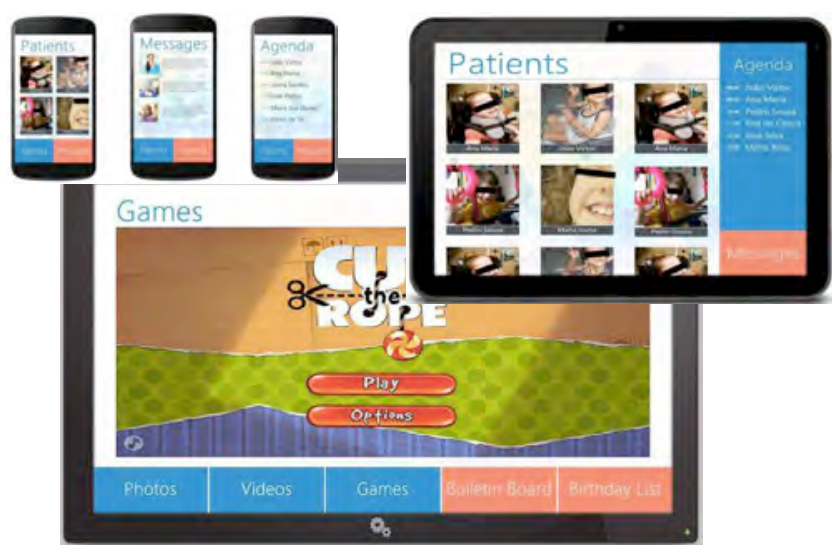

Figure 3: Upper right corner shows smartphone displays, upper left is tablet display and lower image is of CareHub content on the large display. Of particular note is that the smartphone shows private information, but not intended for editing; the tablet is the primary means for data organization and entry while the large displays are for public information and content for the residents. [24]

shows examples of the content of the CareHub for the different platforms.

At this stage, we have deployed the prototype using an intranet that we installed and are collecting data about its usage. Preliminary indications are promising. Initially, we started with five tablets that we supplied and one large display. After 3 weeks of deployment, there were already requests for two personal cellphones to have the prototype installed and one additional tablet for using the system. The system is also designed with a web interface so that any smart device with a browser can use the messaging capabilities. We do not know yet how many people are using this feature. One other incident that indicate that staff are adopting the prototype was one of our students who maintains the equipment got a call at 6am from a staff member indicating something was wrong with the large display (the computer had crashed so was showing the bluescreen of death). The student indicated how to restart the computer over the phone and eventually the system was back to normal, much to the expressed relief of the staff member. This suggests that they are already depending upon some of the functionality. Finally, we recorded 250 messages over a four-week period from 20 staff demonstrating they have been using it without intervention from us. This has also occurred during a strike at the center, so there is a reduced staff count as well as a substantial additional workload on them, yet they are still using it.

\section{TICKET SYSTEM: VISUALIZING FAVORS}

As discussed in [7], we also investigated the role of public visualization of workflow data within the Center. We built a task-managing kiosk that allowed healthcare professionals to post tasks, or mark existing tasks as resolved, in order to compare two graphical representations of tasks. The kiosk consisted of a 17" display with a mini-pc and a wireless 
access point attached to the back of the display. A Portuguese QWERTY keyboard and mouse were provided as peripherals to input text and provide point-and-click interaction (Fig. 5-A, 5-B). The software consisted of a web-form to submit a message up to 250 characters in length, a drop-down list containing all employees of the care unit, a drop-down list containing previously submitted and unresolved tasks, and a submit button (Fig.5-C). A list of unresolved (Fig. 5-D) and resolved (Fig. 5-E) tasks was shown chronologically on the right side of the screen. A visualization (Figure 5-F) of tasks resolved was presented as the main focus of the application. The interaction flow consisted of users selecting their name from the name dropdown list, and either selecting an unresolved task from the unresolved tasks drop-down menu to mark it as resolved, or typing a task to be added to the unresolved list of tasks. The kiosk was installed in the nursery room of the care unit, close to the paper-based log-books historically used by healthcare professionals to delegate and track important tasks. (Fig. 4)

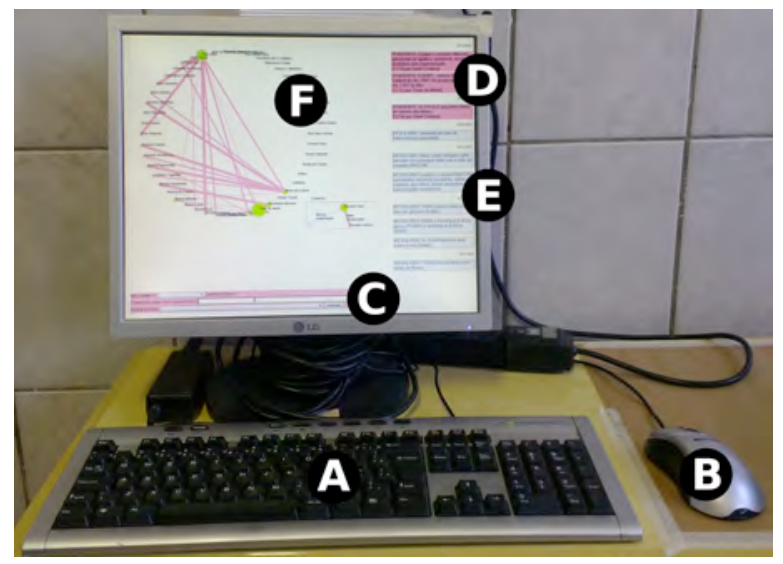

Figure 4. Task-managing kiosk showing a communitycentric visualization of tasks resolved by the community.

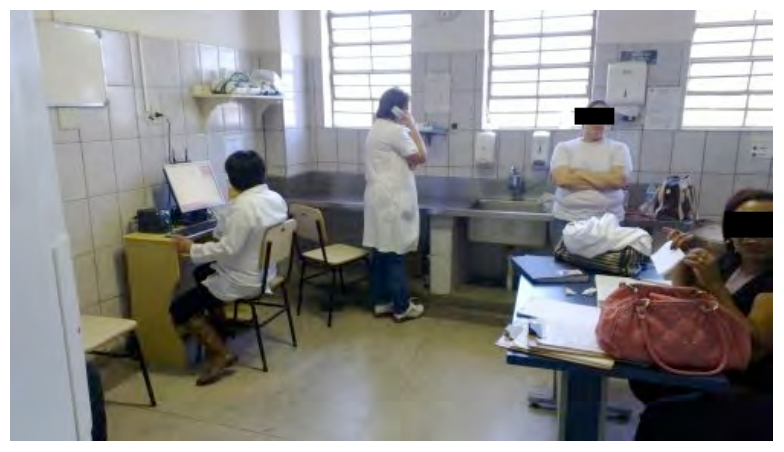

Figure 5. The kiosk installed in the nurses room and being used by one nurse, while others perform daily activities.

Two graphical representations of tasks were made public during one month each as shown in figures 6 and 7. The first visualization (Fig. 6) represented people as circles whose radii depended on the count of resolved tasks. The second visualization (Fig. 7) represented people as circles whose radii represented resolved tasks in relationship to the community, i.e. normalized by total number of tasks resolved. In the latter visualization, lines between circles represented reciprocated actions (i.e. you resolved a task I posted, I resolved a task you posted) whose thickness visualized reciprocity rate.

To evaluate the impact of the visualizations, discussed below, we measured the number of messages posted as well as reciprocity. We used a reciprocity measure that calculated how many times a person who provided a favor had their favor attended to [10].
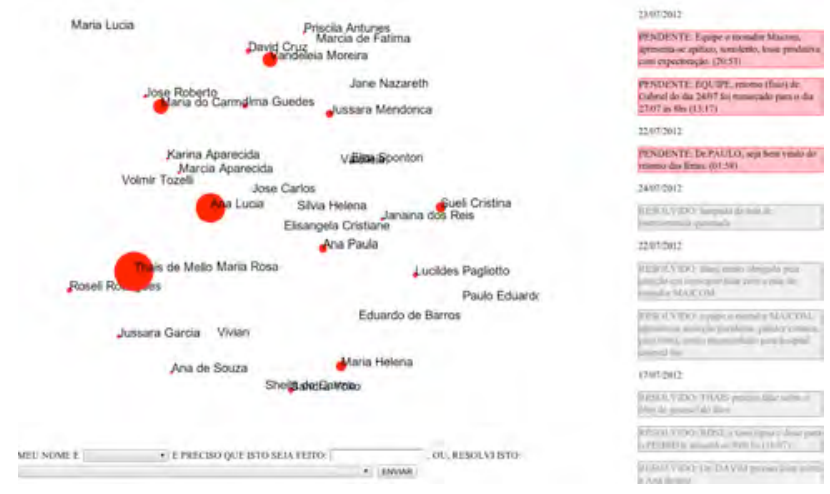

Figure 6: Individual-centric visualization showing people as isolated circles with the size of the circle being related to how many tickets they resolved.
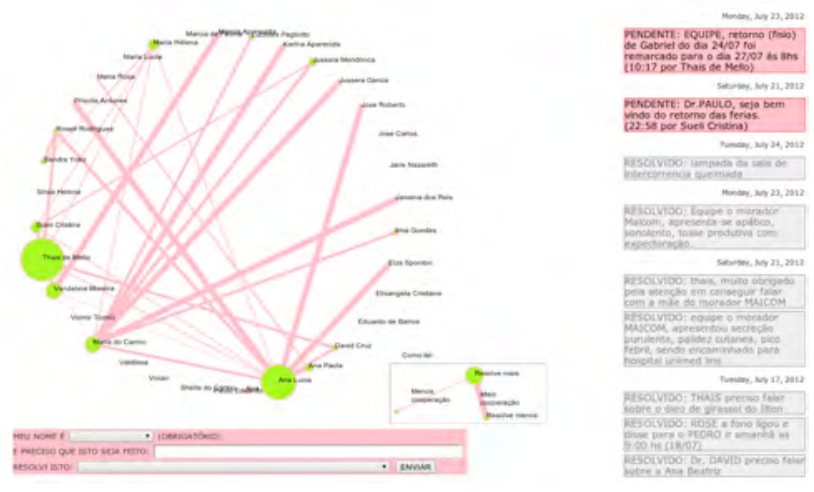

Figure 7: Community-centric visualization showing circles whose radius and connections depict people in relationship to the community or other people. (Screen-shot taken with all tasks collected during all 8 weeks.)

\section{LESSONS LEARNED}

In the following sections, we describe the three main findings thus far at the Center.

\section{Social Intranet}

Initial results using the CareHub show promise for our approach to develop tools and systems oriented towards the healthcare worker to aid them in managing their personal work activities and assets. We are continuing to evaluate 
the effectiveness of our approach and will be taking the feedback from the healthcare workers to design the next iteration of prototype that will more closely match their routine. The staff have been very enthusiastic about this direction even though it has very little administrative mechanisms, such as meeting organization tools, patient health record access, or location services. This is in contrast to the common logbooks at the nursing stations that they are supposed to use to track patient health. These are intended to both keep a log of the patients as well as communicate between staff shift changes any important information. These tend not to be used, but instead, during shifts, just a verbal discussion passes the information around. We believe that ultimately, the personal nature of the approach we are developing for the workers can open the door to include some administrative elements as well as supporting adoption of patient-centred technology that coordinates with the CareHub approach.

\section{Appropriation and Adoption}

Our initial perspective working with the Center was that we were going to look for Natural User Interface (NUI) solutions [29] for their information problems impacting their ability to help patients transition. While this approach is consistent with contemporary UCD design of emerging technologies, we quickly realized that these design methodologies were missing an important component for this context. More specifically, we observed that the community of healthcare workers regularly are problem solving by appropriating the tools and resources around them. The dynamic nature of the environment does not lend itself to a "solution" but rather, an assemblage of resources that can be re-purposed when and where needed. This was their natural workflow and so we redefined our research goals with the Center to focus on how to integrate mobile, consumer grade, ICT into their current natural work rather than finding best practice use of the technology. This approach gave rise to two new main research challenges: 1 . what is a design process to follow for maintaining natural workflow and 2. What is natural and how to measure it.

To address the first point, our proposed design process [2], shown in figure 8, includes both adoption and appropriation activities. We consider adoption as the process of users learning and accepting a designed solution for some of their activities. In contrast, we look at appropriation as the use of an artifact designed for one type of problem, to solve a separate one. We arrived at this process by looking to define design for natural processes to have two properties: 1 . the introduction of technology should not interrupt existing workflow and 2. that the adopted technology must also be shown to be appropriated for use in other contexts. To accommodate these properties, in our HCI design process, we added an additional phase to the usual design-prototype-evaluate design process [20]. From our observations designing for the Center, we determined that it is necessary to support users to first pass through the adoption phase so that they perceive the affordances of a developed solution or prototype. Then, it is important to include affordances and mechanisms for supporting the appropriation phase where they can envision the use of such a solution in a new context.

Thus, if we reconsider our development process, we begin with a Construction phase triggered by some initial requirements. We envision this phase including established HCI user-centered development (UCD) processes such as covered in [20]. Traditionally, the criteria for successful design in the construction phase for WIMP interfaces focuses on usability and usefulness to improve the chances that an artifact will be adopted by the target user community for a specified set of tasks. For a Center context, researchers $[5,16,21,18]$ have used a 3-phase design-prototype-evaluate approach. For example, we employed participatory design; ethnography, work place shadowing and other UCD design strategies during our design activities at the center.

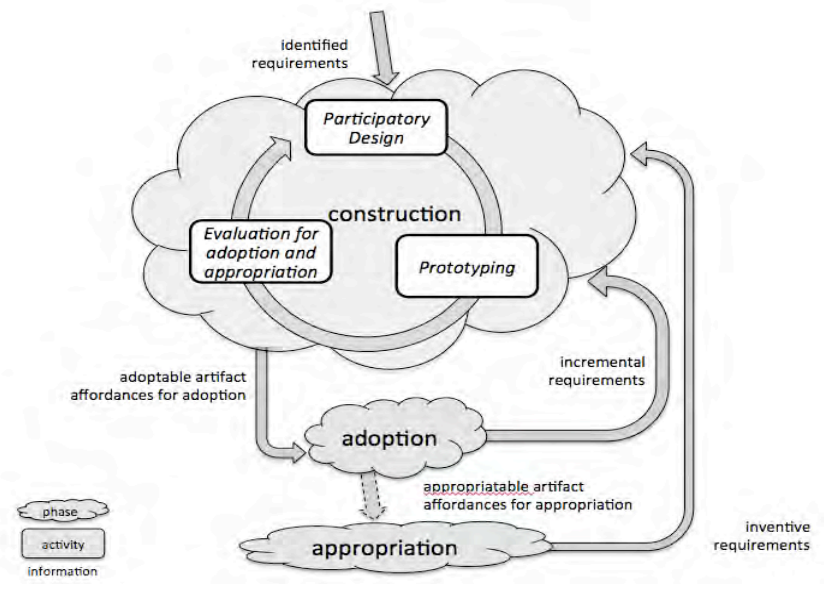

Figure 8: Natural ICT Design Process. Considering the cultural context, appropriation can occurs and naturally is considered a requirement for the next design cycle, naturally integrating cultural values to the solution

Each iteration within the Construction phase leads to a release of an ICT artifact. Once the artifact is created, the next step is deployment and adoption by the target user group. This could be either a small test group or the expected target population. Once adopted, new requirements arise from the users to improve the system to solve the problem that the system was designed for. These normally come as incremental requirements to adjust the interaction design to allow for a gradual refinement to a well-designed system to solve user's problems that they can adopt easily. The systematic adoption evaluation activity in the construction phase attempts to discover these requirements to increase the effectiveness of adoption. In UCD, this process continues from the early stages of construction to the complete lifecycle of the product. As 
shown in figure 1., this is the process we initially performed to find "solutions".

Our observations suggest that while the process, up to this point, leads to establishing new methods to iteratively improve adoption of solutions to a particular problem, it does not include any method to facilitate appropriation. Thus, we add the appropriation phase as part of the development cycle for maintaining natural workflows that incorporate the type of problem solving we observed. During the adoption phase, a user (or possibly users) becomes comfortable enough with the technology that he or she may appropriate the design for a new context to solve problems unforeseen during the Construction phase.

Once appropriation occurs, the user creates new requirements based on the new context of use and the appropriated solution. These are inventive requirements. The appropriation evaluation in the Construction phase can then establish these new, inventive requirements to feed into a new design. The design may be an entirely new product tailored to solve the problems in the new context in a new way.

Designing for appropriation remains a challenge. Dix's basic studies on appropriation [8], suggests that designers should accept that we do not understand completely what will happen in real use with ICT solutions. He argues this is especially true within the context of non ICT-based workflow. Nonetheless, based on our Natural Design process, it is necessary that the ICT solutions are designed so that they can be used in unexpected ways. Further, they need to address the natural environment dynamics so that designing for use is designing for change. To accommodate this, we suggest, the construction phase of design should embody the principles for appropriation listed in Table 1 that are refined points from [8]. Dix studied appropriation of mature, domesticated and becoming-obsolete technology, so that users can repurpose and reinvent its use. For our design space where healthcare workers are using mobile technologies, we define principles for appropriation of technologies that are not yet fully adopted as we envisage that designers design for adoption and appropriation in parallel. Thus, the construction phase design process creates artifacts that allow repurposing while users are becoming comfortable enough with the technology. With each iteration of the construction phase the number of users becoming comfortable with the design grows, leading to more opportunities to discover a user finding new uses.

Another perspective on designing for appropriation follows from discussions about the differences between Norman sense of affordances [19] and Gibson's sense [12] [17]. As pointed out in [17], Norman affordances provide visual cues as to the function of a particular interface that match users common sense knowledge of use. This helps the process of adoption and also leads to less learning required. In contrast, Gibson affordances are the latent functions that an object can support. The typical example provided is that a chair has clear visual affordances that it can be used to sit on; a Norman affordance. However, there are other functions, such as standing on, that may not be visually obvious, but that the chair can support. These would be Gibson affordances as the chair affords making the user taller. Typical, WIMP interfaces focus on Norman affordances for their functions but tend not to afford other functions making it difficult for users to appropriate to new contexts.

In our design process, including Norman affordances supports the design for adoption and leads to easier to use interfaces. For the design for appropriation, however, Gibson affordances are important. As a user encounters new contexts in their activities, there is a natural inclination to appropriate objects designed for one purpose for use in the new one. The ability to do this depends upon the object having the potential to be used in the new context and the user to perceive this. By designing Gibson affordances into the artifact so that objects have the potential to be used for different purposes, this natural evolution is supported. Or conversely, part of the measure of naturalness, is whether the designed objects are appropriated for use in contexts other than what the designed Norman affordances are for. This insight is included in our design process in Figure 8.

In summary, our Natural ICT Design process leads to designs that both support adoption and appropriation. For our work, we are applying our approach to gesture-based, mobile solutions with the additional constraint that it should impose minimal disruption in the existing workflow to be natural. However, we suggest that the Natural ICT Design approach is also appropriate for ICT solutions where the aim is to support the natural evolution of work practices.

\section{Visualization of workflow}

As reported in [7], we found that each visualization of tasks promoted different social dynamics within the community and usage of the kiosk. Visualizing people as isolated circles whose radii increased by resolving tasks promoted competition with peers. This visualization also promoted the creation of content that demanded actions from particular individuals or groups.

By contrast, visualizing people as circles whose radii depended on community actions, and evidencing reciprocal actions as lines connecting people, promoted a desire to either belong to the group, or to avoid being perceived as not participating. This visualization resulted in an increase of messages addressed to the whole community, or messages that could be acted upon by anybody that came in contact with the display.

Furthermore, we observed that the flexibility provided by the text input of our prototype allowed users to circumvent the original design of the ticketing system and adapt it to accommodate their existing communication practices, like using hand-typed timestamps, addressing 
particular individuals, and broadcasting messages of sociability and encouragement.

\begin{tabular}{ll}
\hline Principle & Description \\
\hline $\begin{array}{l}\text { Allowing } \\
\text { interpretation }\end{array}$ & $\begin{array}{l}\text { Including elements where users can add } \\
\text { their own meanings }\end{array}$ \\
Providing visibility & $\begin{array}{l}\text { It is often the irrelevant } \text { state and internal } \\
\text { process that can be appropriated. }\end{array}$ \\
Exposing intentions & $\begin{array}{l}\text { Deliberately exposing the intention } \\
\text { behind the system. }\end{array}$ \\
& $\begin{array}{l}\text { Appropriations may subvert the rules of } \\
\text { the system }\end{array}$ \\
& $\begin{array}{l}\text { Designing a system so that the task can } \\
\text { be done instead of a system to do the } \\
\text { task. }\end{array}$ \\
Supporting, not \\
controlling
\end{tabular}

Table 1. Principles for Designing for Appropriation (modified from [13] and appears in [2])

We believe that both of the tested visualizations had benefits on how people approached their workplace tasks. Visualizing individuals as separate entities strengthened existing ties and supported addressing particular individuals with concrete tasks. Meanwhile, a visualization making evident the social ties between members of the community enticed healthcare professionals to interact with a wider range of individuals through open-ended messages and undirected requests.

We observed that the community slowly adapted the finished prototype to suit their own well-established information and communication management practices. Addressing tasks to individuals or adding task deadlines (features that were not elicited during the user-centred design process) became new practices after some individuals introduced them. This observed adaptation of a task-publishing system to support socialization and personal interaction was unplanned by the researchers, but reinforced the need to include socialization according to the cultural values as a core requirement for future technology to be introduced within the studied community.

\section{CONCLUSION}

We are investigating how to introduce ICT into a long-term mental care institution in Brazil that currently does not use ICT tools in their workflow. Consistent with the trends for developing countries, we are focusing on personal consumer grade mobile devices [14]. Working closely with this group we have reoriented our thinking from designing best practice ICT solutions to providing community support tools and systems that can be appropriated easily to solve problems that come up on a daily basis and require ingenuity by the staff to solve. We argue this behavior for long-term care is common in developing economies who are appropriating personal devices for improving their workplaces rather than wait for enterprise health information systems more common in developed economies. Thus, for these environments, we argue that UCD should include an additional loop focused on appropriation. Appropriation design success can be measured by how little impact the technology has on existing workflow and at the same time, how many new uses and context of use users find for the design. As part of this strategy, we developed a social intranet and a workflow ticketing tool. The ticketing system was appropriated to become a general messaging tool. We are still investigating the online social intranet design to support the staff's mental model of work as a social activity, however, the requests by staff for us to provide it on personal devices indicates adoption is underway.

\section{ACKNOWLEDGMENTS}

We thank the staff at the Center for their participation in this research. We thank our funders: FAPESP, MITACS, NSERC, CAPES/DFAIT, NCE GRAND, Microsoft, and Boeing. We appreciate our colleagues helpful discussions from OCADU and Dalhousie University. We work under the Center Ethical Statement.

\section{REFERENCES}

1. Alankus, G, et al. Towards customizable games for stroke rehabilitation. SIGCHI'10, 2010.

2. Anacleto, J. C. ; Fels, S. Adoption and Appropriation: A Design Process from HCI Research at a Brazilian Neurological Hospital, INTERACT2013.

3. Anacleto, J.; Fels, S.; Silvestre, R.; Souza Filho, C.E.; Santana, B. Therapist-centred design of NUI based therapies in a neurological care hospital. In: SMC2012. Korea, 2012.

4. Anacleto, J.C. 2011. UNITY Project. http://lia.dc.ufscar.br/UNITY/overview.html.

5. Bath, P. A., Health informatics: current issues and challenges. J. Inf. Sci. 34-4, pp. 501-518, (2008).

6. Broderick M., Smaltz D., HIMSS E-Health White Paper: e-Health Defined. [accessed 2011 Nov 17]: http://www.himss.org/content/files/ehealth_whitepaper. pdf, (2008). 
7. Calderon, R.; Fels, S.; Anacleto, J., Towards Supporting the Existing Workplace Practices of a Community of Brazilian Healthcare Professionals, INTERACT'2013, pp. 689-696, 2013.

8. Dix, A. Designing for appropriation. BCS HCI (2): 2730, (2007).

9. Doyle, J., Kelly, D., Caulfield, B. Design considerations in therapeutic exergaming. Pervasive Computing Technologies for Healthcare (PervasiveHealth). 2011; 389-393.

10. Garlaschelli, D., and Loffredo, M. I. Patterns of Link Reciprocity in Directed Networks. Phys. Rev. Lett. 93 (2004).

11. Geurts, L, et al. Digital games for physical therapy: fulfilling the need for calibration and adaptation. Int Conf on Tangible, Embedded, and Embodied interaction, p.117-124,2011.

12. Gibson, James J., The Ecological Approach to Visual Perception. New Jersey, USA, Lawrence Erlbaum Associates, (1979).

13. Greenberg, S, et al. The narrative storyboard: telling a story about use and context over time. Interactions . 2012; 19(1).

14. Junglas, I. and Harris, J., The Promise of Consumer Technologies in Emerging Markets, CACM, vol 5(56), pp. 84-90, 2013.

15. Lange, B., et al. Development and evaluation of low cost game-based balance rehabilitation tool using the microsoft kinect sensor. Eng. Medicine and Biology Society. 2011.

16. Li J., Wilson L., Stapleton S., Cregan P. Design of an advanced telemedicine system for emergency care. OZCHI '06 Proceedings of the 18th Australia conference on Computer-Human Interaction: Design: Activities, Artefacts and Environments, pp. 413-416, (2006).

17. McGrenere, Joanna and Ho, Wayne, Affordances: Clarifying and Evolving a Concept. In: Proceedings of Graphics Interface 2000 May 15-17, 2000, Montreal, Quebec, Canada. pp. 179-186, (2000).

18. Norman, D.A. Natural User Interfaces Are Not Natural. Interactions, 17(3):6-10, (2010).

19. Norman, Donald A., Affordances, Conventions, and Design. In Interactions, 6 (3) pp. 38-41, (1999).
20.Preece, J., Rogers, Y. and Sharp, H., Interaction Design. John Wiley \& Sons, Inc., New York, NY, USA, (2002).

21. Reeder B., Turner A. M., Scenario-based design: A method for connecting information system design with public health operations and emergency management, Journal of Biomedical Informatics archive, V. 44 Issue 6, p. 978-988, 2011.

22. Reeder, B, et al. Reusable design: A proposed approach to Public Health Informatics system design. BMC Public Health 2011, 11:116. 2011.

23. Schönauer, C., et al. Chronic pain rehabilitation with a serious game using multimodal input. Virtual Rehabilitation (ICVR), 1-8, (2011).

24. Silvestre, R.; Anacleto, J. and Fels, S., Designing a Healthcare Worker-Centred System for a Chronic Mental Care Hospital, HWID Workshop INTERACT2013, 1-6, 2013.

25. Sutcliffe, A, et al. User engagement by user-centred design in e-Health. Philosophical Trans. Royal Society A: Math, Physical and Eng. Sciences, v.368, i1926.2010; 4209-4224.

26. Sutherland, M, Maiden, N. Storyboarding Requirements. IEEE Software. 2010;27(6):9-11.

27. Tanaka, K., et al. A Comparison of Exergaming Interfaces for Use in Rehabilitation Programs and Research. Loading.... 2012; 6(9), pp. 69-81.

28. Vegoda, P. Introduction to hospital information systems. IJ Clin Monit Comput. 1987.

29. Wigdor, D and Wixon, D., Brave NUI World: Designing Natural User Interfaces for Touch and Gesture, Morgan Kaufmann, 2011. 